\title{
Research and development of the classification algorithm based on the method of reference planes
}

\author{
Goshin Ye.V., Loshkareva G.E., \\ Samara State Aerospace University \\ Fursov V.A. \\ Samara State Aerospace University \\ Image Processing Systems Institute, Russian Academy of Sciences
}

\begin{abstract}
In this paper a classification algorithm for hyperspectral images based on the reference planes using the values of the contingency table is developed and researched. We propose a new procedure for generating the reference planes. The training vectors areformed with the use of vectors from other classes. The results of experiments on the test image are given.
\end{abstract}

Keywords: hyperspectral images, method of reference planes, classification, conjugacy indices

Citation: Goshin Ye.V., Loshkareva G.E., Fursov V.A. Research and development of the classification algorithm based on the method of reference planes. Proceedings of Information Technology and Nanotechnology (ITNT2015), CEUR Workshop Proceedings, 2015; 1490: 304-308. DOI: 10.18287/1613-0073-2015-1490-304-308

\section{Introduction}

Thematic classification of hyperspectral images is gaining popularity. Studies in remote sensing are useful in many fields such as agriculture, mineralogy, physics, surveillance, forensics etc. Remote sensing of the Earth surface allows us to survey the productivity of the lands, forest fires, construction of roadsand various objects.

The most widely used algorithms of thematic classification for solving the above problems are based on methods of spectral angle and support vector machine (SVM). This paper presents a classification algorithm based on the method of reference planes using the values of the conjugacy indices.

\section{Statement of the problem}

The source data is a hyperspectral image obtained by remote sensing [1]. It is a data structure in which intensity values are stored with the coordinates $X, Y, \mathrm{Z}$, which includes spatial coordinates $(X, Y)$ and spectral coordinates $(Z)$. The objective is to find the given object in this hyperspectral image. 
It is assumed that each of spatial coordinates of the hyperspectral image belongsto one of $K$ classes. Each class represents some object (for example, field of corn, field of wheat, roads, etc.).

\section{Description of the classification algorithm}

To describe the algorithm, consider the $k^{\text {th }}$ class. In this class we will choose the reference plane $X_{k}$ [2] consisting of a pair of hypervectors. For selected reference plane $k$ class index of conjugacy [3] with the vectors from other classes can be calculated with the following expression:

$$
R_{k}(j)=\frac{\mathbf{x}_{j}^{T} \mathbf{Q}_{k} \mathbf{x}_{j}}{\mathbf{x}_{j}^{T} \mathbf{x}_{j}}
$$

where $\mathbf{Q}_{k}=\mathbf{X}_{k}\left[\mathbf{X}_{k}^{T} \mathbf{X}_{k}\right]^{-1} \mathbf{X}_{k}^{T}, \mathbf{x}_{j}$ is vector $j$ from the class $n \neq k, j=1, \ldots, N_{n}$. Thus, we have $N_{n}$ values of conjugacy indices $R_{k}$. For these indices the root-mean-square (RMS) value can be calculated as following

$$
\mathbf{R}_{\text {mean }}(n)=\sqrt{\frac{\left(R_{j}\right)^{2}}{N_{n}}}
$$

where $n=(1, \ldots, K) \backslash k$. Next, we do the same with the remaining classes. For all remaining $K-1$ values $\mathbf{R}_{\text {mean }}$, we calculate RMS value

$$
\mathbf{R}(i)=\sqrt{\frac{\left(\mathbf{R}_{\text {mean }}(n)\right)^{2}}{K-1}}
$$

where $n=(1, \ldots, K) \backslash k, i=1, \ldots, C_{N_{n}}^{2}$.

Further, we similarly define conjugacy indicesfor all planes $X_{k}$ of $k^{\text {th }}$ class and calculate $C_{N_{n}}^{2}$ values of $\mathbf{R}(i)$, where $i=1, \ldots, C_{N_{n}}^{2}$. Then the minimum value $\mathbf{R}(i)$ is sought among them

$$
R=\underset{i=1, C_{N_{n}}^{2}}{\min } \mathbf{R}(i) \text {. }
$$

The hyperplane which corresponds to the received minimum value $\mathbf{R}$ is stored. Let us denote it as $\mathbf{Y}_{k}$. Thus, we get the reference plane with the highest value of the conjugacy index with its class. This plane allows us to effectively recognize the chosen class. At the same time, it will be less responsive to vectorsof other classes. This allows us to enhance the effectivity of the hyperspectral images classification. 
On recognition stage [4] it is necessary to choose the minimum threshold of conjugacy. It is performed by calculating the conjugacy indiceswith the reference plane $\mathbf{Y}_{k}$

$R_{Y}(m)=\frac{\mathbf{x}_{m}^{T} \mathbf{Q}_{Y} \mathbf{x}_{m}}{\mathbf{x}_{m}^{T} \mathbf{x}_{m}}$,

where $\mathbf{Q}_{Y}=\mathbf{Y}_{k}\left[\mathbf{Y}_{k}^{T} \mathbf{Y}_{k}\right]^{-1} \mathbf{Y}_{k}^{T}, \mathbf{x}_{m}$ is vector $m$ from class $k, m=1, \ldots, N_{k}$, and $N_{k}$ is the number of vectors in class $k$. The $N_{k}$ values obtained from this procedure are sorted in ascending order. Then first (lowest) or $\mathrm{N}^{\text {th }}$ value from ordered sequence of $R_{Y}$ is chosen.

\section{The results of the experiment}

The algorithm was tested on a Salinastest image from open dataset of hyperspectral image MultiSpec. This image was obtained within AVIRIS program (AirborneVisible/ InfraredImagingSpectrometer). The image size is $512 \times 217$ hyperpixels. Each hyperpixelhas 224 spectral bands. A sample of hyperspectral layer and classified test image are shown in figures 1a), 1b), respectively.

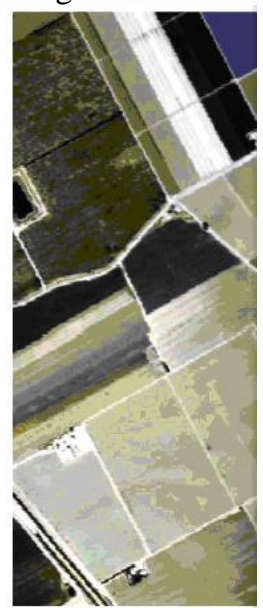

a)

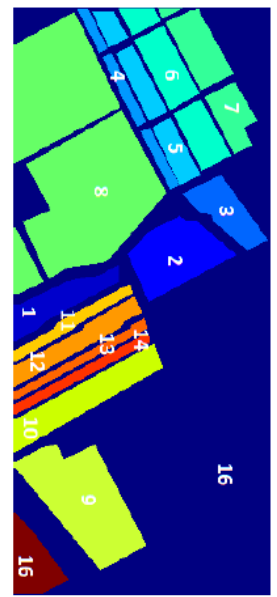

b)

Fig. 1. - Salinas test image: a) hyperspectral layer with the use of artificial coloring; b) classified image

The algorithm was applied to detect the 4th, 5th, and 11 th classes (the area with roughlyplowed fields, smooth ground, four-week lettuce, respectively). The figure shows the location of the fifth and eleventh class vectorswith skipping the $20 \%$ lowest conjugacy indices. The figuresshowsa few falsely recognized hyperpixels from other classes. 

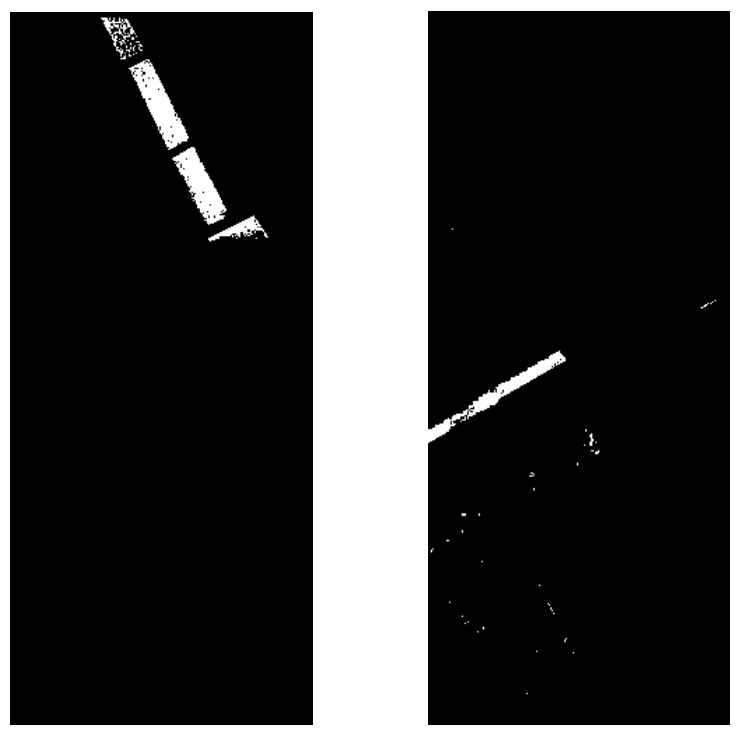

Fig. 2. - Location of 5 and 11 classes vectors respectively

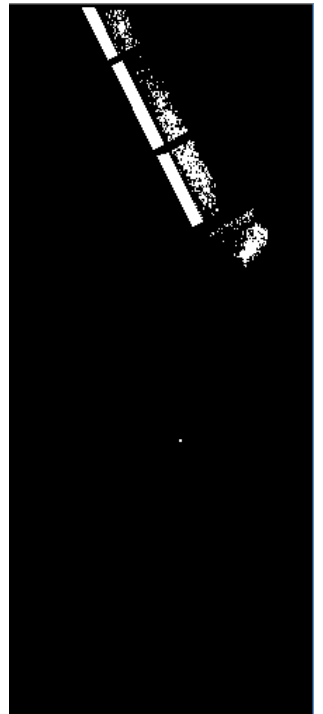

a)

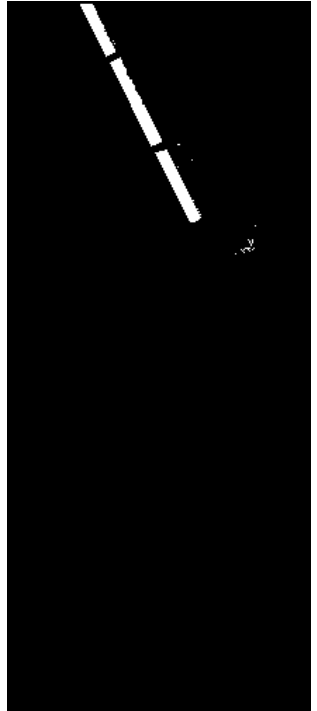

b)

Fig. 3. - Location of the fourth class vectors: a) the $0 \%$ threshold; b) the $2 \%$ threshold

Figures 3a), 3b) shows an example of the recognition of the fourth class vectors with a different minimum conjugacy threshold. The figures show that the choice of the threshold strongly influences the number of recognition errors inother classes. The increase in the threshold enhances recognition quality in its class. 


\section{Conclusion}

The choice of an appropriate reference plane in the proposed algorithm has a high computational complexity. Recognition quality depends on the choice of the minimum conjugacy threshold. A small value of the threshold increases the number of recognition errors associated with "wrong detection" from other classes. The increase in the threshold leads to thepoints loss in a recognized class.

\section{Acknowledgements}

The work was supported by Ministry of Education and Science of the Russian Federation (project No. 2930).

\section{References}

1. Lowengart RA. Remote sensing. Models and methods for image processing. Moscow: Technosphere, 2010; $560 \mathrm{p}$.

2. Zherdev DA, Kazanskiy NL, Fursov VA. Object recognition by the radar signatures of electromagnetic field scattering on base of support subspaces method. Computer Optics, 2014; 38(3): 503-510. [in Russian]

3. Fursov VA. Training in Pattern Recognition from a Small Number. 15th International Conference on Pattern recognition, 2000; 2: 119-121.

4. Fursov VA, Bibikov SA, Baida OA. Thematic classification of hyperspectral images using conjugacy indicator. Computer Optics, 2014; 38(1): 154-157. [in Russian] 